\section{Space constraints}

Rev. Sci. Instrum. 82, 023901 (2011)

The majority of artificial satellites circulate in a low Earth orbit - that is, between 200 and $1,000 \mathrm{~km}$ altitude. There they have to cope with a harsh environment: orbital debris and micrometeorites tend to get in their way, but there is also considerable radiative and chemical stress on the satellite structures. The degradation of the materials that make up the satellites is evaluated typically by retrieving samples and inspecting them back on Earth. Ronen Verker, Eitan Grossman and Irina Gouzman now propose a method for measuring the degradation of 'space materials' in orbit.

There are certain devices that are part of every satellite: photovoltaic cells. Verker and colleagues propose covering several of these cells with different semitransparent materials, while having other, uncoated cells for reference. The differences in output power will then depend on how much the covering material has been degraded.

In a simulated low-Earth-orbit environment, Verker et al. tested a range of films and obtained encouraging results. Amorphous carbon, for example, turned out to be sensitive to fluxes of atomic oxygen, whereas in cells coated with a material known as Kapton the output was also correlated with morphology changes.

\section{Positive discrimination}

Phys. Rev. Lett. 106, 062301 (2011)

Brookhaven's Relativistic Heavy Ion Collider (RHIC), and now CERN's Large Hadron Collider, facilitate the study of the very hot, dense phase of matter thought to have existed in the early history of the Universe. Data from RHIC have suggested charge asymmetry fluctuations among the particle debris of the heavy-ion collisions something is happening to cause an uneven distribution of electric charge. A possible culprit is the chiral magnetic effect (CME): solving the Dirac equation in a magnetic field reveals that the lowest Landau level is chiral; if some of these levels are filled, then a current is induced.

If the CME is proved to be at work in the RHIC data, then this would be a direct observation of a topological effect in quantum chromodynamics, and indicate that chiral symmetry is restored in the plasma. But the picture is blurred by the likely presence of a chiral vertical effect (CVE), arising from the combination of vorticity and baryon chemical potential.

Dmitri Kharzeev and Dam Son think there is a way to tell the two apart. It means measuring, event by event, the baryon asymmetry - the distribution of threequark particles formed in the aftermath of the collisions - as well as the charge asymmetry. The magnitude of their ratio, which should increase as the centre-ofmass energy decreases, is quite different (by an order of magnitude) for the CME and the CVE.

\section{In a trap, but not alone}

Nature doi:10.1038/nature09721 (2011)

Nature doi:10.1038/nature09800 (2011)

Quantum mechanical oscillators, such as ions caught in a trap, are one possible building block for quantum computers. Controlled coupling between two such systems enables the transfer of information between them, a vital process in quantum information processing. Such coupling has now been achieved independently by two groups of scientists.

Two ions trapped on a chip can interact with one another via the Coulomb effect: the closer the ions, the stronger the interaction. However, concentrating traps into a very small area leads to problems caused by heating, particularly once you take into account the effect of all the electrodes that are needed.

Kenton Brown and colleagues counteract this heating by cooling their ion 'chip' to $4.2 \mathrm{~K}$ in liquid helium. They observed energy transfer between two beryllium ions separated by $40 \mu \mathrm{m}$. Max Harlander and co-workers, on the other hand, used auxiliary trapped ions as an antenna to boost the interaction. In this way they were able to couple calcium ions over a distance of $54 \mu \mathrm{m}$.

\section{The corona also rises}

Astrophys. J.Lett. 729, L8 (2011)

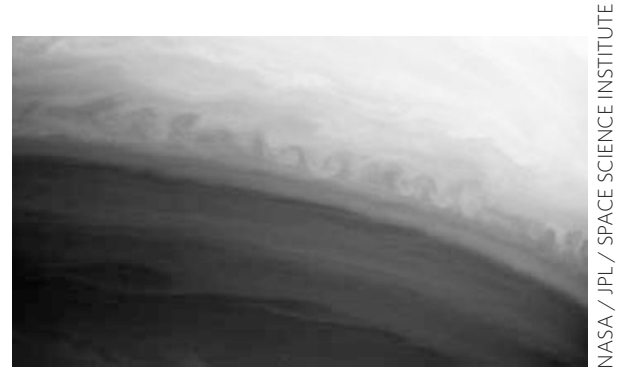

Coronal mass ejections release blasts of plasma and radiation from the Sun's corona. Particularly strong shock waves can damage satellites and bring down power grids on Earth, or allow Bostonians to read the newspaper at night by auroral light alone. In fact, the Sun will soon come out of its quiescent phase and our ability to predict solar activity will determine how prepared we are for any potential damage. Fortunately, the Solar Dynamics Observatory was launched in March 2010.

Studying images taken by the on-board Atmospheric Imaging Assembly, Claire Foullon and co-workers noticed some familiar wave patterns in images taken in the extreme ultraviolet band, at 11 million Kelvin. Just as two fluids flowing at different velocities lead to Kelvin-Helmholtz instabilities at the interface (like these ones, pictured, in Saturn's atmosphere), there are whirls with associated wavelength $\left(18 \times 10^{6} \mathrm{~m}\right)$ and speed $\left(833 \mathrm{~km} \mathrm{~s}^{-1}\right)$ in one flank of the ejecta. The presence of these instabilities will help researchers understand the kinematics of coronal mass ejections and the local nonlinear dynamics that drive them. 